\title{
De gré ou de force : le microcrédit comme dispositif néolibéral
}

Cyril Fouillet (Essca), Isabelle Guérin (IRD-Cessma, Institut Français de Pondicherry), Solène MorvantRoux (University of Genève) et Jean-Michel Servet (IHEID)

Version du 18 janvier 2016

Fouillet C., Guérin I. Morvant-Roux S. Servet J.-M. (2016) De gré ou de force : le microcrédit comme dispositif néolibéral, Tiers Monde, 225 (1) : 21-48.

Résumé

Le secteur du microcrédit a fait face à plusieurs crises d'impayés ces dernières années. A partir d'enquêtes menées en Inde, au Maroc et en République dominicaine, cet article montre que ces crises n’ont en rien ébranlé l'un des mythes à l'origine de ces crises : la croyance en les capacités autorégulatrices par le marché. L'objectif de croissance reste prioritaire (même si c'est à des degrés divers selon les contextes) et se traduit à la fois par des pratiques actives de démarchage (il ne s'agit plus de répondre à une demande, mais de la créer) et une focalisation sur un segment finalement très restreint de la clientèle, celle qui a déjà fait ses preuves. Une lecture de ces crises en termes d'économie politique permet également de resituer le microcrédit dans l'évolution des politiques dites de « développement » axées sur l'inclusion financière. En normant les comportements des populations, le microcrédit apparât alors comme révélateur d'un dispositif néolibéral au sens de Michel Foucault.

Mots-clés : crise du microcrédit, dette, développement, inclusion financière, Inde, Maroc, microfinance, microcrédit, néolibéralisme, République dominicaine, surendettement.

\section{Introduction}

Espace structuré et microcosme inclus dans l'espace global du développement, le champ de la microfinance offre un terrain d'observation privilégié des enjeux liés aux dynamiques contemporaines des pratiques monétaires et financières.

Dans cet article, la description des fortes montées d'impayés dans différentes régions de la planète, principalement entre 2007 et 2011, subies par les institutions de microfinance (IMF) délivrant des microcrédits (Guérin, Labie et Servet 2015) ${ }^{1}$ constitue notre point de départ pour développer dans un second temps une lecture en termes d'économie politique du microcrédit comme révélateur d'un dispositif

\footnotetext{
${ }^{1}$ Le microcrédit est défini ici comme la provision de crédit à des personnes non incluses dans le système bancaire. Ce terme est souvent confondu avec celui de microfinance, qui fait référence à la provision d'un ensemble plus vaste de services financiers : du microcrédit mais aussi des produits d'épargne, d'assurance, de facilités de transferts, etc. Dans l'article, lorsqu'il est fait mention de crises, celles-ci font référence au microcrédit et aux défauts de paiement. Cependant, lorsque nous nous référons aux organisations délivrant ces microcrédits, nous utilisons le terme d'institution de microfinance (IMF).
} 
néolibéral ${ }^{2}$. Une lecture classique du champ de microcrédit fait apparaitre une relation structurante dans laquelle s'affrontent, d'un côté, les tenants d'un microcrédit ne poursuivant que des objectifs de rentabilité financière, et, de l'autre, ceux à la recherche d'un impact social en termes de réduction de la pauvreté et des inégalités (Morduch, 2000 ; Woller et al., 1999). Les observations conduites sur nos trois terrains d'enquête permettent de dépasser cette opposition en resituant le microcrédit dans l'évolution des politiques dites de « développement » axées sur l'inclusion au marché (market based pro-poor development policies) (Berndt, 2015 ; Johnson et Williams, 2013).

Pour atteindre cet objectif, l'article reprend les résultats d'enquêtes menées par les auteurs en Inde, au Maroc et en République dominicaine, et réalisées en collaboration avec d'autres chercheurs ${ }^{3}$. Ces enquêtes ont pris en compte les actions et les points de vue des acteurs tout au long de la chaine de production et de distribution des services financiers, ainsi que leurs compréhensions et leurs usages par les destinataires finaux.

\section{Répondre à une demande ou la créer ?}

Le traitement des dernières données mises à disposition par le Microfinance Information eXchange Market (MIX Market), malgré certaines limites ${ }^{4}$, permet d'observer l'évolution de l'offre de microcrédit depuis le milieu des années 1990. D’un point de vue global, une période de très forte croissance peut être identifiée jusqu'en 2010, suivie d'une diminution du nombre d'emprunteurs entre 2010 et 2012 en raison principalement - mais pas seulement - de la chute du portefeuille de plusieurs IMF indiennes liée à la crise dans l'État de l'Andhra Pradesh (voir fig. 1 et 2). Depuis, la croissance de l'offre est de retour. Toutefois, selon les informations diffusées par la Campagne des sommets du microcrédit (Reed, 2014), le nombre de clients pauvres, en valeur absolue et relative, continue de baisser ${ }^{5}$.

Malgré ce ralentissement de l'expansion du secteur, très inégalement réparti selon les pays (Guérin et al., 2015), nombre de promoteurs du microcrédit considèrent toujours que le potentiel d'expansion du microcrédit est considérable. Dans The Porffolio of the Poor, ouvrage de référence auprès des praticiens et des chercheurs du secteur, les auteurs de ce best seller affirmaient que «le microcrédit au Sud représente le plus

\footnotetext{
2 Dans son cours donné au Collège de France entre janvier et avril 1979 et intitulé Naissance de la biopolitique, Michel Foucault (2004) analyse le retournement qu'a opéré le néolibéralisme par rapport au libéralisme. L'État, loin d'être rejeté, est mobilisé pour permettre le bon fonctionnement de la concurrence ainsi que la normalisation et la discipline des individus afin d'y être soumis avec le maximum de consentement.

${ }^{3}$ Les données mobilisées dans cet article ont été récoltées dans le cadre du projet « Microfinance in crisis » financé par la Banque Européenne d'investissement (BEI). Ces enquêtes combinent analyse statistique et analyse compréhensive. L'analyse statistique s'appuie sur des enquêtes ménages longitudinales menées par les auteurs (Inde), ou des enquêtes de systèmes d'information et de gestion d'IMF partenaires (Maroc et République dominicaine). L'analyse compréhensive s'appuie sur des entretiens avec une diversité d'acteurs (clients, non clients, agents de crédit, responsables de branches ou d'IMFs, prêteurs privés, etc.) et des observations directes, en particulier concernant l'interaction entre agents de crédit et clients. Ces données ont été collectées depuis 2002 en Inde, en 2008 au Maroc, et plus récemment (2013) en République dominicaine. Pour plus de détails, voir Isabelle Guérin et al. (2015) et www.microfinance-in-crisis.org.

${ }^{4}$ Même si une partie des IMF de petites tailles ne déclarent par leurs activités au MIX Market, cette base reste représentative dans la mesure où le secteur de la microfinance se concentre essentiellement sur de grandes IMF, toutes présentes dans ces données.

${ }^{5}$ Selon les données du rapport 2014, le nombre de clients très pauvres serait passé de 138 à 116 millions entre fin 2010 et fin 2012. Le nombre de clients total atteignait environ 200 millions fin 2012.
} 
grand marché au monde » (Collins et al., 2010). Investisseurs et cabinets de consultants, dont le rôle est croissant dans ce qui est aujourd'hui devenu une véritable industrie, concordent grosso modo dans leurs estimations : 1,5 milliard de personnes auraient besoin de microcrédit (Hes et Poledňáková, 2013), alors que 200 millions seulement en bénéficient. Ces estimations font écho à un large pan du courant dominant de la recherche sur le « rationnement » du crédit ( $f$. notamment l'article séminal de Stiglitz et Weiss, 1981), facteur supposé de «sous-développement » et de pauvreté (voir, par exemple, Banerjee et Moll, 2010).

Figure 1: Nombre d'emprunteurs et d'épargnants sur le marché mondial de la microfinance (en millions) (1996-2013)

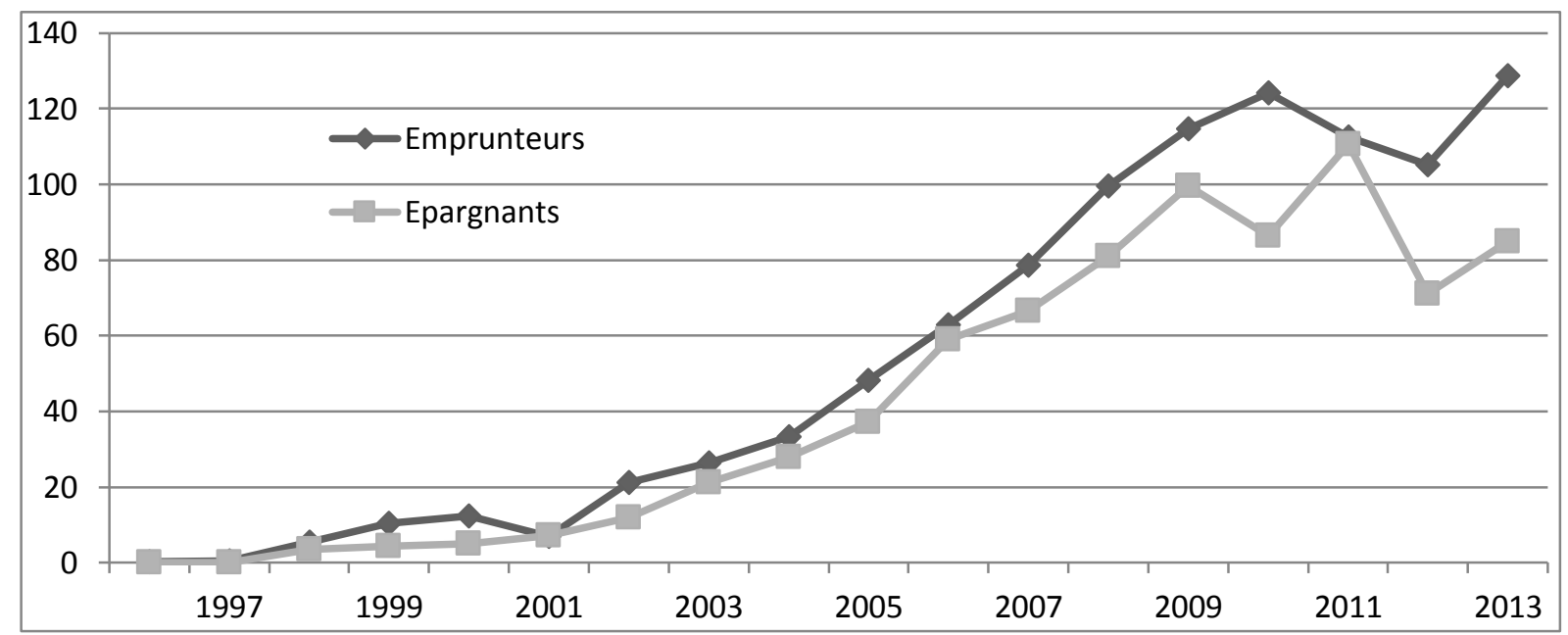

Sources : données compilées d’après la base MIX Market.

Figure 2: Nombre d'emprunteurs sur le marché de la microfinance en Inde (en millions) (1996-2015)

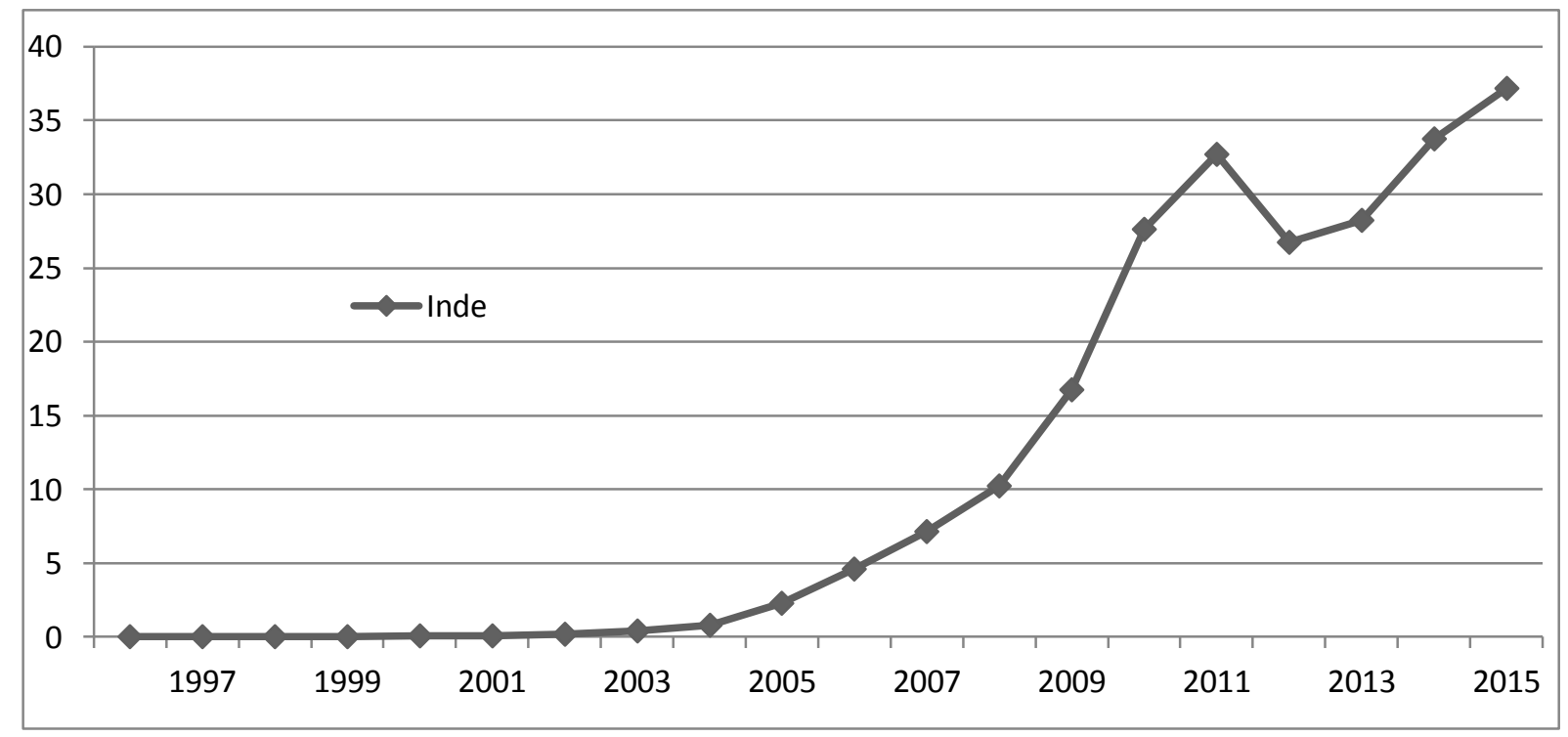

Sources : données compilées d’après la base MIX Market. 
Des analyses empiriques montrent que tant la demande effective que les capacités locales d'absorption du crédit sont en réalité largement surestimées. Il s'agit d'ailleurs de l'un des principaux résultats des études d'impact randomisées. À défaut de pouvoir véritablement expliquer l'impact des prêts (Bédécarrats et al., 2015 ; Bernard et al., 2012 ), ces expérimentations ont pour point commun de pointer une adhésion " modeste » des populations au microcrédit et des écarts entre estimations initiales et taux constatés de participation (Banerjee et al., 2015 ; Morvant-Roux et al., 2014). Lorsqu'il n’y a aucune présélection, le taux de participation varie entre 17 et $31 \%$ (ibid., p. 10) : autrement dit, entre 83 et $69 \%$ de la population cible ne veut pas de microcrédit.

\section{Figure 3: Nombre d'emprunteurs sur le marché de la microfinance au Maroc et en République dominicaine (en centaine de milliers) (1997-2014)}

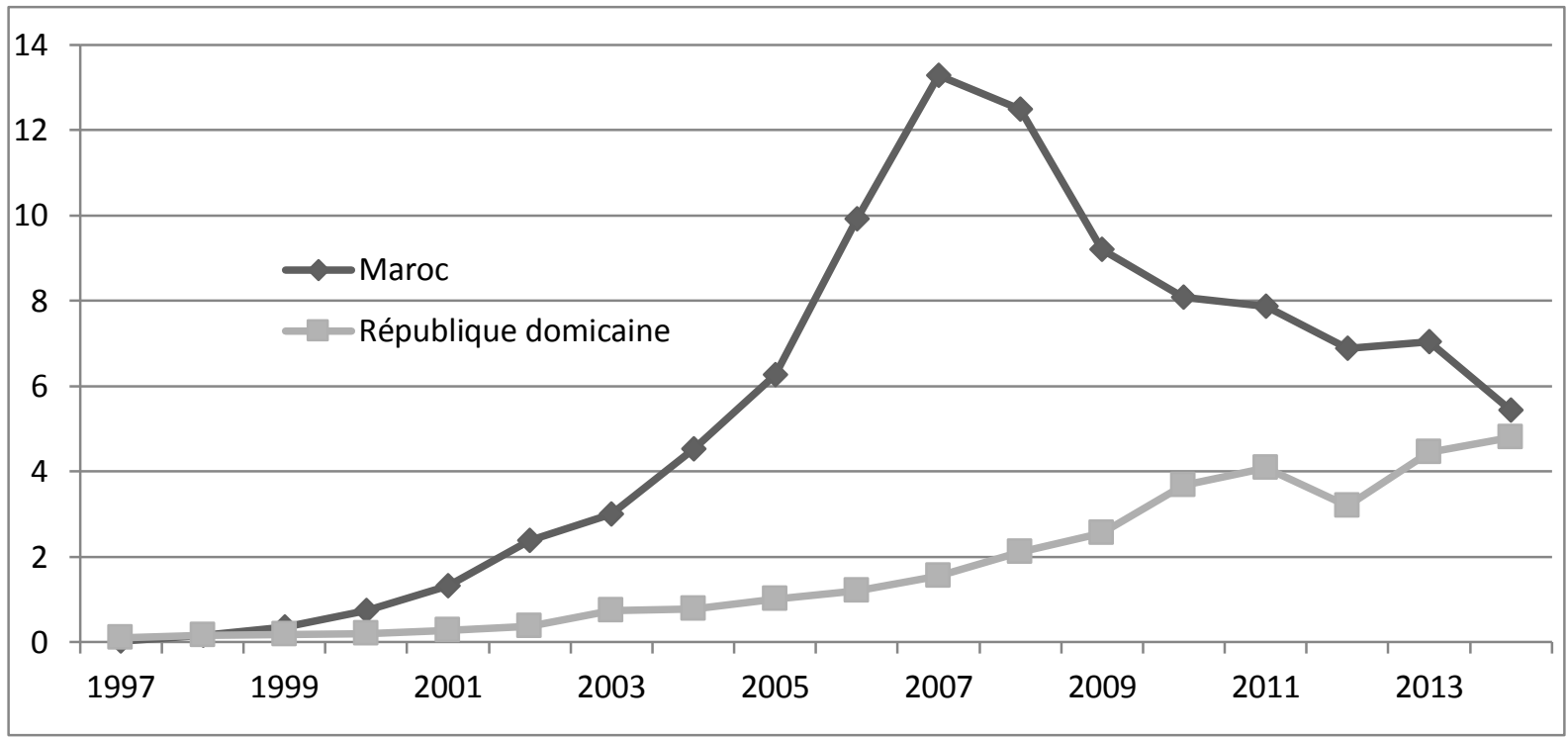

Sources : données compilées d'après la base MIX Market.

Dans les trois terrains étudiés ici, après une période de forte croissance, un tassement peut être observé pour chacun des pays et, dans certains cas, un recul du nombre d'emprunteurs actifs (figures 2 et 3). Le cas du Maroc est tout particulièrement intéressant. L'effondrement brutal qui suit 2009 s'explique par la faillite de Zakoura, l'un des principaux acteurs du secteur. Cependant, la reprise du portefeuille de clients de Zakoura par Attawfiq (émanation de la Fondation Banque populaire) n'a pas permis de compenser plusieurs années de baisse. En 2014, le marché de la microfinance marocain retrouvait son niveau de 2004, soit une baisse de $59 \%$ par rapport au pic atteint en 2007 avec plus de 1,3 million d'emprunteurs. En Inde, le regain de croissance observé en 2013 s'explique principalement par le déploiement de certaines IMF dans des régions où la microfinance était jusque-là absente. Les IMF situées dans le Sud de l'Inde, en Andhra Pradesh ou au Tamil Nadu, connaissent des difficultés fortes et leur légitimité auprès des populations a diminué.

Doit-on en conclure que le modèle du microcrédit tel qu'adopté par les principales IMF observées dans ce projet arrive à saturation ? Les données récoltées dans le cadre du projet « Microfinance in Crisis » et dont une partie est présentée dans les sections suivantes inclinent à le penser. 


\subsection{Inde, Tamil $\mathrm{Nadu}^{6}$}

Les enquêtes de terrain menées par l'équipe de l'Institut français de Pondichéry depuis 2002 font apparaître un surendettement chronique des populations rurales de cet État indien. D’après nos estimations, entre un cinquième et un quart de la population est pris dans un cercle vicieux de surendettement, au sens où la dette est un facteur d'appauvrissement plus que de stabilisation des revenus ou d'accumulation (Guérin et al., 2012 ; Guérin et al., 2013). Les populations n'ont pas attendu le microcrédit pour s'endetter, et le surendettement observé reflète des tendances structurelles bien plus étendues. S'entremêlent ainsi une forte propension à l'endettement - pour des raisons socioculturelles -, une dépendance croissante à l'égard du salariat urbain - mais qui reste journalier ou à la pièce et sans aucune protection ni régularité -, et enfin des aspirations croissantes qui se traduisent par des investissements sociaux conséquents, notamment en termes de rituels sociaux et religieux, d'éducation et d'habitat.

Il n'est donc pas surprenant de constater que la distribution de crédits domestiques au niveau national a connu une forte hausse dans les années 2000. Les crédits dédiés à l'achat de biens immobiliers de la part de particuliers, de biens durables ainsi que les prêts à la consommation ont triplé entre 2001 et 2009 (fig. 4). Dans l'État du Tamil Nadu, sur la même période, ces prêts ont été multipliés par six ou presque (480\%). Au-delà d'une diffusion plus importante des prêts pour des usages « personnels », ce sont aussi les territoires qui sont repensés. Tandis que les zones rurales étaient délaissées depuis la libéralisation du secteur en 1991 (Landy, 2001), on observe depuis 2010 un intérêt croissant des banques commerciales privées pour ces territoires considérés comme de nouveaux marchés. La ICICI Bank, première banque commerciale privée du pays, connue depuis sa création en 1994 pour être une banque de la nouvelle classe moyenne, investit désormais les campagnes. Entre 2010 et 2014, sur l'ensemble du territoire indien, le nombre de ses agences rurales a quadruplé. En 2014, dans l'État du Tamil Nadu, ICICI Bank comptait 363 agences dont 127 localisées dans des villages sans autres entités bancaires. Les IMF ne sont donc pas les seules pourvoyeuses de crédits sur le sol indien, loin s'en faut, et l'agressivité des banques commerciales publiques comme privées ne doit pas être sous-estimée ; les nouveaux intermédiaires commerciaux appelés Business Correspondent, dont l'objectif est de créer un lien entre les zones délaissées, le secteur bancaire et les transferts sociaux de l’État sont là aussi pour placer des crédits.

\footnotetext{
${ }^{6}$ Les données utilisées dans cet article pour les IMF indiennes proviennent du MIX Market, tandis que les données sur les Self-Help Groups (SHG) sont issues de rapports de la Nabard, la Banque nationale indienne pour le développement rural et agricole. Au 31 mars 2014, $68 \%$ des microcrédits octroyés aux SHG provenaient d'une banque commerciale, à $26 \%$ d'une Regional Rural Bank et à $6 \%$ d'une banque coopérative. Les données utilisées pour le secteur bancaire indien ont été compilées à partir des rapports de la Reserve Bank of India (RBI). Les IMF avec lesquelles nous avons travaillé sont BWDA, Equitas, KalviKendra, KCRDS, Smile, PMSS dans les districts de Villipuram, Cudallore, Tiruvallur, Vellore et dans l'État de Pondichéry. Concernant le nombre d'entretiens, compte tenu de la période d'observation (2002-2014), il est difficile de donner une estimation : plusieurs centaines d'entretiens ont été menés. À cela s'ajoutent plusieurs enquêtes longitudinales avec des échantillons comprenant 280 à 400 familles. Pour plus de détails, voir Isabelle Guérin et al. (2015).
} 
Figure 4 : Évolution du nombre de prêts personnels distribués dans l'année par les banques commerciales indiennes (en millions, à gauche) et évolution du nombre d'agences rurales (en milliers, à droite) (2001-2014)

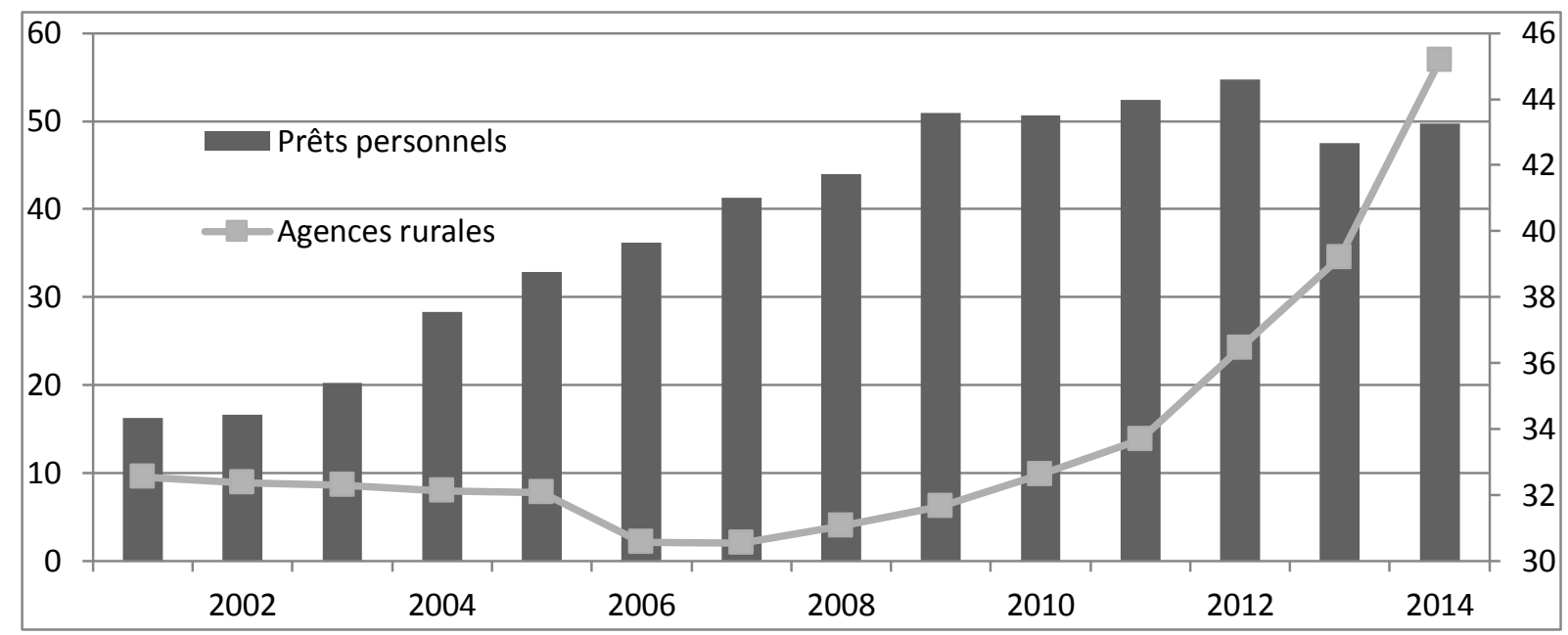

Sources : compilation à partir des données de la RBI.

Compte tenu de la multiplicité des sources d'endettement et de la complexité des chaînes de causalité, quantifier le rôle du microcrédit est certainement un exercice vain. On peut néanmoins raisonnablement affirmer qu'il y contribue, pour deux raisons : des procédures d'octroi déconnectées de la solvabilité réelle des emprunteuses, et des pratiques de recouvrement très rigides qui s'accommodent mal de revenus irréguliers et parfois imprévisibles. Ceci a été largement développé ailleurs (Guérin et al., 2009, 2015). Nous insisterons simplement ici sur la manière dont les IMF réagissent aujourd'hui à la saturation du marché. Plutôt que d'identifier de nouvelles recrues, il leur est plus facile de se focaliser sur des clients existants et de les encourager à emprunter toujours davantage. Les données disponibles tant pour les IMF que pour les Self-Help Groups (SHG) au niveau macro illustrent ce phénomène (fig. 2 et 5). Si l'on se focalise sur les SHG indiens, le nombre de prêts octroyés à ces groupes de femmes a considérablement diminué depuis la crise de l'Andhra Pradesh, passant de 1,6 à 1,2 million (fig. 5). En revanche, le montant moyen des prêts ne cesse d'augmenter : entre 2007 et 2014, celui-ci a été multiplié par trois, passant de 60000 à 180000 INR7 (de 833 à 2500 euros, soit un montant moyen par membre d'environ 60 à 178 euros). Même en tenant compte de la hausse des prix, cette augmentation apparaît considérable. Ces données générales dissimulent d'autres tendances. Tout d'abord, l'arrivée dans le secteur de la microfinance indienne de nouveaux acteurs ouvertement tournés vers les prêts à la consommation. L'IMF Muthoot, par exemple, dont les activités de microcrédit ont été lancées en 2010, en pleine crise du secteur, affiche depuis un taux de croissance annuel moyen de $87 \%$. Ensuite, tandis que plusieurs IMF historiques connaissent des difficultés importantes depuis 2010, telles Basix ou Sarvodaya Nano Finance Limited, d'autres IMF ont rapidement repris le chemin de la

\footnotetext{
${ }^{7}$ Roupie indienne.
} 
croissance. Dans le Tamil Nadu, on peut citer les cas d'Equitas, d'Arsivad ou d'Evangelical Social Action Forum, par exemple.

Figure 5 : L'offre de microcrédit en Inde. Évolution du nombre de prêts octroyés aux SHG durant l'année (en milliers, à gauche) et évolution du montant moyen des prêts (en milliers de roupies, à droite) (1993-2014)

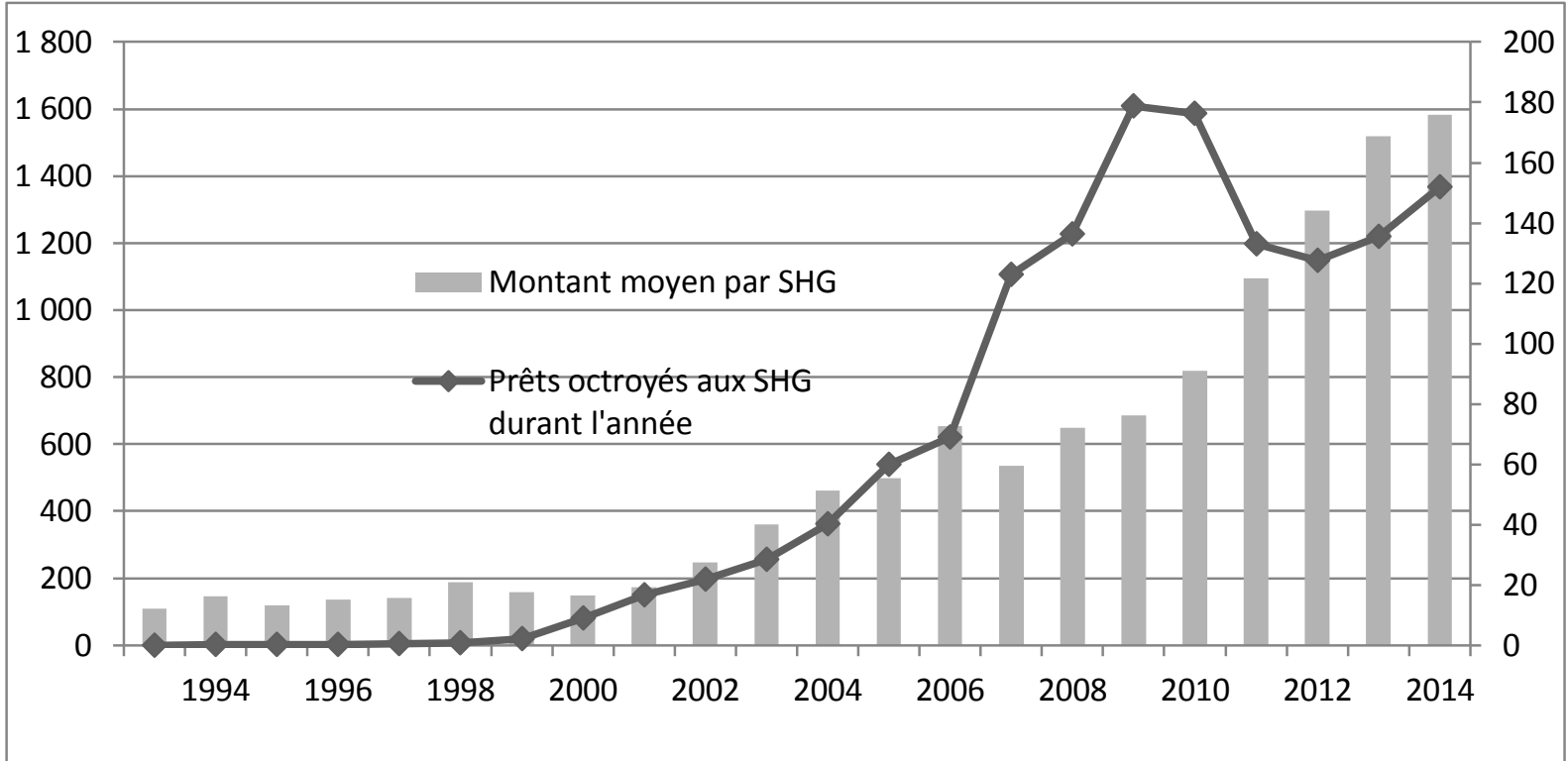

Sources : compilation à partir des rapports annuels de la Nabard.

Cette tendance est largement confirmée par des observations qualitatives. Les femmes mentionnent à quel point il leur était difficile d'accéder au crédit autre qu'informel il y a une dizaine d'années et combien elles ont l'embarras du choix désormais. Les plus gros montants sont supposés dédiés à des activités génératrices de revenus, mais ceci est une illusion. Du côté de l'offre, les responsables de branche et les agents de crédit sont également très explicites : la crise de l'Andhra Pradesh, très largement couverte par les médias, a été une opportunité pour écarter les groupes à risque, privilégier les plus performants et en contrepartie augmenter les montants des prêts. Depuis 2011, suite à la crise, la législation impose aux IMF de nouvelles conditions : plafonds de prêts basés sur les revenus des emprunteurs, restrictions quant à l'usage dédié à la consommation - qui est supposée représenter moins de $30 \%$ du prêt -, enregistrement dans une centrale de prêt et flexibilité dans les remboursements. Dans le même temps, pour diverses raisons liées notamment à la volonté de l'État du Tamil Nadu de reprendre le contrôle des activités de développement, de nombreuses organisations non gouvernementales $(\mathrm{ONG})$ de microcrédit ont fermé leurs portes alors que d'autres acteurs sont apparus : des ONG reconverties en compagnies financières non bancaires et des compagnies financières qui offrent désormais aussi une composante microcrédit ${ }^{8}$. Parmi les IMF avec lesquelles nous

\footnotetext{
${ }^{8}$ On observe également ces phénomènes dans le reste de l'Inde. C'est par exemple le cas de l'IMF Fusion Microfinance créée en janvier 2010 ou de SV Creditline, une compagnie financière proposant depuis 2010 des microcrédits et affichant des taux de croissance spectaculaires : le nombre de clients est passé de 10000 à 143000 de mars 2010 à septembre 2013.
} 
avons travaillé, à l'exception des prêts croisés, que certaines mentionnent, les autres conditions ne sont pas respectées, voire souvent ignorées par les agents. Difficilement compatibles avec la nature de la demande principalement orientée vers la consommation, ces conditions apparaissent aussi incompatibles avec les contraintes de croissance. Comme l'exprimait un agent de crédit, le principal enjeu de leur métier est de « saisir le marché » (catch the market).

Par contre, les prêteurs informels sont incapables de suivre cette fuite en avant. La taille de leur portefeuille est réduite (parmi ceux rencontrés, les plus importants ne dépassent pas quelques centaines de clients) et leurs perspectives de croissance restent limitées, à la fois du fait de contraintes de financement et de difficultés pour identifier et contrôler des clients quand ils deviennent très nombreux.

Combien de temps cette fuite en avant dans ce secteur du crédit se poursuivra-t-elle ? Le système paraît être soutenable tant que la croissance économique se maintient et qu'elle offre des perspectives d'emploi, permettant ainsi d'absorber le coût du crédit. Migration de courte durée et programmes d'emplois publics ${ }^{9}$ sont les stratégies les plus fréquentes de remboursement. Mais la croissance s'essouffle (avec un taux actuel annuel de croissance du produit intérieur brut (PIB) de 3-4 \% contre 9-10 \% jusqu'en 2010). Faute de mécanismes de protection sociale et compte tenu de la précarité et de l'irrégularité qui caractérisent les relations d'emploi, l'offre de microcrédit contribue à une économie d'endettement paraissant peu soutenable à moyen et long termes.

\subsection{République dominicaine ${ }^{10}$}

Le choix de la République dominicaine peut sembler incongru au vu de l'apparente bonne santé du secteur de la microfinance dans ce pays. Banco Adopem et Banco Ademi figurent entre autres parmi les IMF les mieux notées et primées au monde pour leur professionnalisme ; l'application des Best practices du CGAP (Consultative Group to Assist the Poor) ou encore la standardisation de la formation des agents de crédit (Afonso, 2013). À cela s'ajoute la présence de deux centrales de risques privées particulièrement opérationnelles et couvrant l'essentiel de l'offre, y compris, pour l'une d'entre elles, une partie - certes réduite - des prêteurs informels (González et Servet, 2014). Banco Ademi et Banco Adopem, qui concentraient près de $70 \%$ des clients du pays en 2014, présentent des portefeuilles « sains » (portefeuille à risque - PAR - à 30 jours inférieur à $3 \%$ ) et figurent ainsi parmi les « bons élèves » d'un secteur fortement incité à se professionnaliser.

Cette situation apparemment sous contrôle dissimule néanmoins des tensions du fait d'une forte concurrence liée à une concentration urbaine très prononcée et de l'entrée de nouveaux acteurs : banques commerciales, banques microfinancières, ONG, programme de crédit public (Banco Reservas), etc. À l'instar de l'Inde, la présence d'une large gamme de prêteurs informels (des petits prêteurs à l'échelle du

\footnotetext{
${ }^{9}$ Depuis 2008, dans le cadre du National Rural Employment Guarantee Act, l'État indien garantit à tout foyer vivant en zone rurale cent jours de travail manuel non qualifié rémunérés. L'ouverture d'un compte bancaire auprès d'un Business Correspondant est souvent un prérequis pour bénéficier de ce programme d'emplois publics.

10 Outre l'analyse des systèmes d'information et de gestion des IMF partenaires (Adopem et Fondesa), l'analyse compréhensive mobilisée ici s'appuie sur 71 entretiens approfondis (47 clients et non clients, 11 agents de crédit, 5 responsables d'agence, 4 responsables d'IMF et 4 prêteurs privés). Pour plus de détails, voir Solène Morvant-Roux et al. (2015).
} 
quartier - appelés san - ou de l'usine, jusqu'aux prêteurs établis ayant pignon sur rue et enregistrés auprès de l'une des centrales de risques) combinée à une société de consommation omniprésente caractérisent un contexte général qui incite à l'endettement.

Nos observations indiquent que le microcrédit, tout en permettant à certaines personnes de stabiliser une activité entrepreneuriale (néanmoins souvent très fragile), affaiblit la situation financière d'une partie de la clientèle. Les objectifs ambitieux de croissance des IMF sont fixés en fonction d'un potentiel à atteindre (besoins non couverts), mais sont essentiellement réalisés sur un segment étroit et saturé du fait de la concurrence. Pour les agents de crédit, remplir ces objectifs de portefeuille est d'autant plus crucial que les incitations salariales sont claires : dans l'une des deux principales IMF de République dominicaine, la moitié du salaire en dépend (Afonso, 2013).

Dans ce contexte, les agents combinent différentes stratégies pour forcer la main aux clients, en « créant les besoins ", comme nous le disait l'un d'entre eux, c'est-à-dire en usant de techniques commerciales pour éveiller l'intérêt du client à (re)prendre d'autres crédits ou à s'endetter davantage (Morvant-Roux et al., 2015). Il s'agit par exemple de renouveler le crédit dès lors que $75 \%$ du précédent a été remboursé pour éviter que le client ne se tourne vers les concurrents. Les agents de crédits rencontrés consacrent une certaine énergie à convaincre les « bons clients » d'emprunter à nouveau. Ceci suppose une démarche active visant à suggérer l'usage de l'argent prêté pour rafraîchir la peinture intérieure, changer le téléviseur ou acquérir un ordinateur pour les enfants : «Quand les gens nous disent qu'ils n'ont pas besoin d'un prêt, nous essayons de les convaincre que ce serait utile. » Sur les cinq agents rencontrés, tous admettent qu'une partie de leur activité consiste à persuader les clients de l'intérêt de s'endetter. Être « bon client» suppose avant tout un historique de crédit positif et sans entraves, que ce soit avec l'IMF ou ses concurrentes. Nos observations montrent que parmi les "bons clients", certains sont en grande fragilité financière, pris dans un cercle vicieux de surendettement, mais ils tiennent néanmoins à respecter scrupuleusement leurs engagements.

Le refinancement de dettes contractées auprès d'autres IMF semble être monnaie courante. La plupart des gestionnaires et des agents de crédit rencontrés nient le pratiquer, mais font valoir que leurs concurrents le font. Pour les agents de crédit, cette stratégie agressive permet de gagner des parts de marché et d'atteindre les objectifs qui leur sont fixés. Une stratégie complémentaire consiste à établir des partenariats avec des entreprises de biens de consommation (réfrigérateurs et écrans plats sont disponibles dans les agences). Cette course au crédit est d'autant plus problématique que les emprunteurs, en cas de difficultés de remboursements, ont peu de marges de manœuvre. Le secteur dispose de deux centrales de risques. Supposées prévenir le surendettement, ces deux organisations jouent en fait un rôle efficace d'incitation au remboursement. Fortement dépendants du crédit, les emprunteurs ne peuvent se permettre d'être classés comme mauvais payeurs et n'hésitent pas à faire des sacrifices multiples pour honorer leurs dettes. Le terme fréquemment utilisé à propos des centrales de risques (El diablo), illustre leur pouvoir coercitif. Plus encore, les agents de crédit utilisent régulièrement les centrales de risques pour démarcher les clients de leurs concurrents.

Soulignons enfin que la concurrence des programmes gouvernementaux est en forte croissance depuis deux décennies, avec la distribution de prêts bonifiés et, d'après les IMF rencontrées, une moindre attention 
aux taux de remboursement. Cette intervention publique est considérée par les responsables des IMF comme une concurrence déloyale et comme un risque potentiel de détérioration de la qualité des remboursements (González et Servet, 2014). Incapables de rivaliser sur les coûts, mais soucieuses de prendre des parts de marché qu'elles ont déjà eu du mal à conquérir, les IMF tentent de rivaliser sur la rapidité d'octroi des crédits. Ceci les amène, là encore, à faire preuve de démarchage actif voire d'agressivité commerciale.

\section{3. $\operatorname{Maroc}^{11}$}

Comparé aux deux contextes précédents, le Maroc se singularise par une faible propension des populations à s'endetter, pour des raisons socioculturelles (Morvant-Roux et al., 2014). Le crédit n'est pas une composante habituelle - et donc nécessaire - de la gestion du budget des ménages, et les formes les plus courantes de la dette sont intégrées aux activités économiques, comme les avances par les fournisseurs largement pratiquées dans les souks (Servet, 2011).

Objectivement, cette faible propension à s'endetter diminue les risques de surendettement. En même temps, et contrairement aux deux contextes précédents qui connaissent un vaste panel d'offres informelles de crédit, la faible présence au Maroc d'« usuriers » les empêche de jouer un rôle d'amortisseur en cas de difficultés de remboursement (Morvant-Roux et Roesch, 2015). Alors que les prêts informels sont très régulièrement utilisés au Tamil Nadu ou en République dominicaine pour rembourser les microcrédits, ce type de pratique est beaucoup plus rare au Maroc. Ceci accentue d'autant la réticence à l'endettement puisque contracter un microcrédit s'avère alors plus risqué.

Cette prudence, observée de manière inégale en fonction des régions, représente un véritable défi pour les agents de crédit : comment trouver des clients pour remplir les objectifs de croissance du portefeuille ? Cette difficulté est renforcée par les tensions liées à un climat post-crise.

En décembre 2009, une crise des impayés secouait l'ensemble des IMF marocaines avec, pour point d'orgue, la faillite de Zakoura, la deuxième IMF du pays, et son absorption par la Fondation Banque populaire, rebaptisée ensuite Attawfiq (IFC, 2014). Cette crise a amené les acteurs à prendre des mesures de court et long termes visant à assainir le secteur (CGAP, 2013).

\section{Figure 6 : Évolution du nombre d'emprunteurs pour les principales IMF marocaines (en milliers) (1997-2014)}

\footnotetext{
11 Outre l'analyse des systèmes d'information et de gestion des IMF partenaires (Attawfiq et AMSSF), l'analyse compréhensive mobilisée ici s'appuie sur 150 entretiens approfondis réalisés au cours de trois missions réalisées depuis 2008 dans différentes régions du Maroc. Pour plus de détails, voir Solène Morvant-Roux et Marc Roesch (2015).
} 


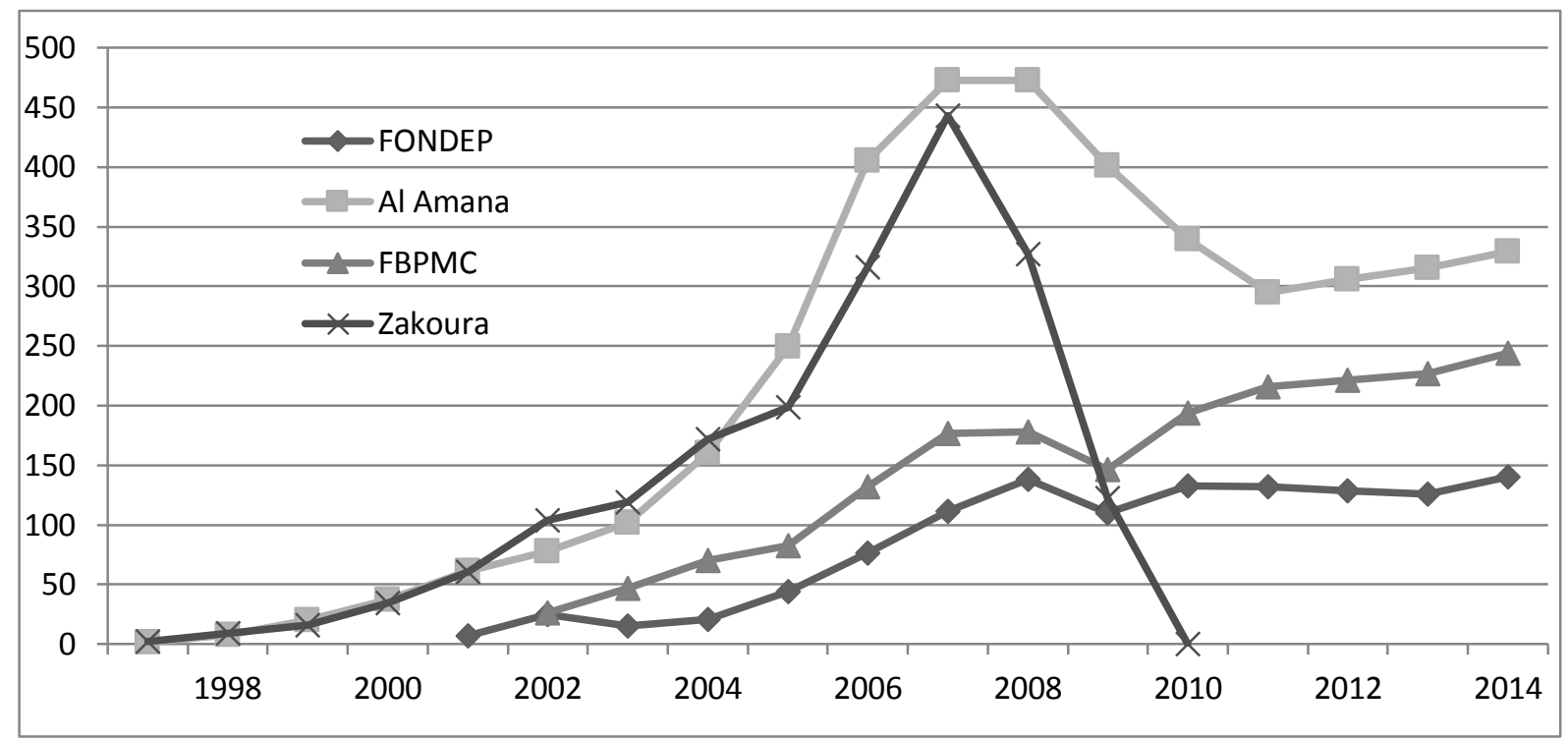

Sources : données compilées d’après la base MIX Market.

Parmi les dynamiques enclenchées dans l'après crise, l'accent a été mis sur l'échange d'information sur les clients via une centrale de risques. Créée en 2009, cette dernière n'est réellement utilisée que depuis 2011. Actuellement, les agences de microfinance sont supposées consulter systématiquement cette base de données, mais, d'après les agents Attawfiq, elle n'est bien souvent renseignée que de façon sommaire (les adresses manquent, les montants prêtés aussi, l'historique des prêts n'est pas à jour, etc.). D’après eux, les IMF ne souhaitent pas fournir ces informations à la concurrence afin que celle-ci ne puisse pas détecter les prêts multiples ou les prêts croisés. Si l'assainissement escompté semble au moins partiellement atteint, nos observations de terrain avec les agents de crédit révèlent pourtant une situation très tendue.

Du côté des clients, la crise du secteur, en particulier la faillite de Zakoura ${ }^{12}$, leur a montré que les impayés (très nombreux chez Zakoura) n'enclenchaient pas les sanctions annoncées par les employés des IMF. Cela a alimenté un sentiment d'impunité que l'on retrouve, par effet de mimétisme, chez la plupart des clients, quelles que soient les IMF.

Ce sentiment d'impunité s'accompagne d'un attachement relativement faible des clients envers les microcrédits proposés par les IMF, pour les raisons mentionnées plus haut. Pour ces deux motifs (faible attachement au crédit, sentiment d'impunité), les incitations à rembourser sont alors limitées, ce qui complique d'autant le rôle des agents de crédit. Non seulement ils peinent à identifier des clients potentiels, mais il leur faut également s'assurer qu'il s'agit de clients fiables. Or, suite à la crise, les exigences du secteur en matière de remboursement sont désormais très élevées et les prises de risque sont minimales. Face à ce dilemme, les agents de crédit ont donc tendance à se concentrer sur les « bons » clients. Tétanisés par la perspective d'enregistrer des impayés et en même temps contraints de respecter une taille minimale de portefeuille, les agents de crédit privilégient leurs relations avec leurs « bons » clients et préfèrent donc

\footnotetext{
${ }^{12}$ Le discours officiel tenu par le personnel consiste à cacher la faillite de Zakoura derrière une simple fusion entre deux institutions. Néanmoins, cette fusion ainsi que le récent changement de nom de la Fondation sont des sources de confusion chez les clients.
} 
augmenter les montants prêtés aux clients fiables plutôt que de prendre des risques avec de nouveaux clients. Quant à l'appréciation des « bons » clients, celle-ci est principalement basée, comme dans les deux autres contextes, sur la «moralité » des personnes et de leur famille et sur leur respect des engagements. Comme ailleurs, les perspectives entrepreneuriales sont limitées, en particulier en zones rurales (Morvant-Roux et al., 2014) : la solvabilité se mesure en termes de bonne réputation puis d'historique de crédit.

D’après nos observations, les agents atteignent $80 \%$ des objectifs quantifiés fixés par leur hiérarchie. Ces résultats ne sont possibles que parce que 80 à $90 \%$ des prêts sont des renouvellements. Il y a très peu de nouveaux prêts. Contrairement au Tamil Nadu, ici, le respect de la procédure et les remboursements l'emportent sur les objectifs. Cette situation se traduit donc par des taux de croissance très modestes depuis 2010, observés pour l'ensemble des acteurs du secteur, et contraste avec les taux vertigineux qui prévalaient avant la crise (D'Espallier et al., 2015). Prenons l'exemple des trois principales IMF marocaines à partir des données du MIX Market. Concernant Attawfiq, le taux de croissance annuel moyen du nombre d'emprunteurs était de 20,4 \% sur la période 2002-2014 et de $6 \%$ entre 2010 et 2014. Pour Al Amana, le taux de croissance annuel moyen entre 2010 et 2015 est légèrement négatif (-0.6\%), alors que la période précédente (1997-2015) enregistrait une croissance annuelle moyenne de $35 \%$. Enfin, la tendance est identique pour Fondep, avec un taux de croissance annuel moyen du nombre d'emprunteurs de 25,8 \% sur treize ans (2001-2014), alors que celui-ci n'était que de 0,11 \% entre 2010 et 2014.

Sur les trois terrains étudiés, on observe en définitive que les objectifs de croissance, difficiles à atteindre même si c'est à des degrés divers en fonction des contextes, se traduisent à la fois par des pratiques actives de démarchage (il ne s'agit plus de répondre à une demande, mais de la créer) et par une focalisation sur un segment finalement très restreint de la clientèle, celle qui a déjà fait ses preuves. Si les critères entrepreneuriaux peuvent entrer en ligne de compte, c'est souvent - et surtout parfois - la moralité des emprunteurs potentiels qui est prise en compte ${ }^{13}$. Simultanément, les besoins de clients considérés moins solvables, plus risqués et moins « disciplinés » sont ignorés. Que les plus pauvres soient exclus leur évite le surendettement, mais ce resserrement pose néanmoins question quant à la fonction originelle du microcrédit.

\section{Les mythes de la régulation par le marché}

L'analyse de la montée des impayés révèle une multiplicité de facteurs (Guérin et al., 2015). Ils s'entremêlent et se combinent, quoique de manière inégale selon les pays et leurs régions : du côté des IMF et de leurs investisseurs, une croissance débridée qui excède les possibilités d'absorption des économies locales ainsi que les capacités de gestion interne des organisations ; du côté des États, une régulation insuffisante ou n'étant pas appliquée, mais également une ingérence pour défendre les emprunteurs qui sont aussi des électeurs potentiels (comme en Andhra Pradesh) (Picherit, 2015 ; Servet, 2011 ; Taylor, 2011) ; enfin, du côté des clients, des formes de résistances organisées, traduisant opportunisme, lassitude ou exaspération

13 Pour des exemples, voir Isabelle Guérin (2015). 
face à l'agressivité des pourvoyeurs de crédits (Guérin, 2014 ; Kar, 2013) et des promesses non tenues, mais aussi, dans certains cas, des intérêts corporatistes liés à des relations clientélistes (comme au Nicaragua).

Dans ce contexte, dont l'un des effets aura été d'avoir atténué l'aura médiatique du microcrédit comme outil essentiel de génération de revenus, un changement de paradigme s'est produit : on est passé d'arguments sur la lutte contre la pauvreté à une légitimation par la nécessité d'une large inclusion financière des populations (Servet, 2015). De nouveaux acteurs ont fait leur entrée : des banques (par le downscaling, c'està-dire la descente en gamme de l'offre de services financiers bancaires) mais aussi des États (pressés par des fondations privées, des firmes privées et des organisations internationales), en développant des programmes d'ouverture de comptes bancaires et en favorisant l'apparition et la diffusion de nouveaux intermédiaires (Fouillet et Morvant-Roux, 2015).

Bien au-delà des terrains étudiés, le surendettement constitue une préoccupation majeure du secteur, comme le dévoilent les deux dernières enquêtes "Banana Skins ${ }^{14}$ » (Lascelles et Mendelson, 2012 ; Lascelles et al., 2014). Quel que soit leur rôle dans le secteur (praticien, investisseur, expert, régulateur ou structure d'appui), toutes les parties prenantes mentionnent le surendettement comme risque premier. Les risques de défaut de paiement et la concurrence viennent en deuxième et troisième places. Les auteurs du rapport « Banana Skins 2014 » indiquent que cette crainte du surendettement est symptomatique de problèmes plus larges : une focalisation sur des objectifs de croissance et de profit au détriment de la prudence, une insuffisance de professionnalisme et une large disposition de fonds à prêter. Globalement - et même si ce constat masque de fortes disparités -, les IMF ont peu de difficultés pour trouver des financements ; elles ont plus de mal à les écouler (Lascelles et al., 2014). Les témoignages des acteurs interrogés, rapportés dans le rapport « Banana Skins », confirment certaines de nos observations de terrain : nouveaux entrants peu soucieux d'éthique, concurrence, absence de centrales de risques, octroi de prêts de montants plus importants, attentes excessives en matière de croissance, comportements « prédateurs », instabilité des revenus des clients, etc.

À l'aide d'autres méthodes (une estimation quantifiée des taux de pénétration du microcrédit comparés à une capacité maximale d'absorption), l'index Mimosa fournit une autre indication des risques de saturation : Bangladesh, Cambodge, Mongolie, Pérou, Arménie et Azerbaïdjan apparaissent comme hypersaturés (Planet Rating, 2013).

Ces observations ont de quoi inquiéter. En préface du rapport « Banana Skins 2014 », le directeur du Centre for the Study of Financial Innovation (CSFI) indique que ces différents constats, en rapprochant le microcrédit de la finance classique, ne doivent pas être interprétés comme un échec de la microfinance : ils attesteraient de sa réussite via sa «normalisation » (Lascelles et al., 2014). Notre position est beaucoup plus pessimiste car il n'y a aucune remise en question sur le paradigme qui a pourtant provoqué les premières crises, à savoir la croyance aux vertus autorégulatrices de la concurrence.

La réponse des acteurs aux risques de saturation du secteur se décline de deux manières. Elle se traduit, d'une part, par l'adoption de «bonnes pratiques » et d’indicateurs supposés juger de la conformité de leurs

\footnotetext{
${ }^{14}$ Depuis 2008, cette enquête est menée régulièrement (à un rythme annuel ou biannuel) et vise à lister les principaux risques perçus par les différents acteurs (praticiens, investisseurs, régulateurs, agences de notation, « observateurs ») du secteur de la microfinance. Elle est menée par le Centre for the Study of Financial Innovation (CSFI), un think tank « indépendant » logé au Centre d'inclusion financière d'Accion, et financée par la fondation Citi.
} 
actions. La croyance aux vertus de l'autorégulation (Bédécarrats, 2013 ; Servet, 2006) demeure, tout en revêtant de nouvelles formes. On trouve ici des indicateurs de performance sociale, des efforts (laborieux) de transparence des prix ${ }^{15}$ et des mesures de protection des consommateurs ${ }^{16}$. D'autre part, on assiste à l'émergence d'une panoplie de mesures d'accompagnement et « d'éducation financière » des clients, mesures supposées leur permettre d'améliorer leurs «capacités financières » (financial capability) et donc de gérer les risques de surendettement. L'éducation financière est l'un des piliers de la Smart Campaign, considéré comme une mesure phare de la protection des consommateurs.

Ainsi, le risque de surendettement est prétendument écarté grâce à des mesures de correction du comportement des emprunteurs via l'« éducation financière » ou encore par des nudges, ceux-ci étant entendus comme des déclics, « des coups de pouce » pour faire adopter à l'individu un comportement supposé adapté et bénéfique à une situation donnée ${ }^{17}$. Resituées dans l'évolution des politiques dites de "développement » axées sur l'inclusion au marché (market based pro-poor development policies) (Berndt, 2015 ; Johnson et Williams, 2013), ces pratiques ne visent plus à corriger les imperfections des marchés ou des politiques publiques, mais les imperfections des comportements.

\section{Pour conclure : le microcrédit (dans sa forme dominante) comme dispositif néolibéral}

Il convient de bien distinguer l'idéologie du néolibéralisme de sa mise en cuvre comme gouvernement des sociétés ${ }^{18}$. Il serait en effet erroné de prendre le discours dominant pour argent comptant et de croire en un recul effectif de l'interventionnisme étatique. Celui-ci a simplement changé de formes et d'objectifs. Il ne vise plus à redistribuer une partie des richesses créées au bénéfice des fractions les plus fragiles de la société et notamment à administrer l'affectation des crédits. De fait, l'État soutient massivement les fractions économiquement et idéologiquement dominantes servant l'utopie d'une autorégulation des systèmes de production et d'échange par la concurrence des intérêts privés ; avec pour conséquence, si l'on y ajoute la défense de la propriété privée et la recherche de la rentabilité financière la plus élevée, une forte concentration des richesses et une croissance des inégalités (voir, entre autres, Lazzarato, 2015). Ainsi, les IMF qui servent les fractions les mieux loties du secteur bénéficient globalement de davantage de subventions que les organisations à statut non lucratif et au service du développement (Cull et al., 2009). Rien d'étonnant, compte tenu des vertus accordées à la concurrence des intérêts, à ce que se développe une agressivité commerciale entre pourvoyeurs de fonds, entre IMF et entre leurs agents de crédits afin d'atteindre les objectifs de croissance des organisations. La concurrence est davantage au service d'une

\footnotetext{
${ }^{15}$ L'ONG MFTransparency, visant à promouvoir la transparence des prix dans le secteur de la microfinance, a œuvré de 2008 à 2014 dans la collecte de données sur les taux d'intérêt payés par les clients. Dans un texte justifiant l'arrêt de ses activités, le directeur de l'ONG considère que son rôle a consisté à alerter le secteur sur la nécessité de faire preuve de transparence et que cette responsabilité incombe désormais aux acteurs. Il mentionne aussi les défis auxquels ils se sont heurtés, compte tenu de la grande réticence des IMF à divulguer leurs tarifs, et en l'absence de législation obligeant à la transparence. Voir Chuck Waterfield, 2015.

16 Procédures d'évaluation (SPI), de certification des IMFS (Smart Campaign, Truelift Pro-Poor Seal of Excellence) ou guide d'action pour les investisseurs (" Principes pour les investisseurs en finance inclusive », élaborés en 2011, qui font partie des « Principes pour l'investissement responsable » des Nations unis).

17 Voir le site http://nudges.org/tag/behavioral-economics/, créé à partir d'un ouvrage de Dean Karlan et Jacob Appel (2011).

${ }^{18}$ Voir note 2.
} 
extension des réseaux que d'une baisse des coûts des services offerts et d'un ajustement aux besoins effectifs des populations (Guérin, 2015 ; Servet, 2015). En retour, les banques de microcrédit, paraissant présenter moins de risques et offrant des normes d'évaluation bien connues des établissements financiers, sont évidemment celles qui sont financées en priorité par les investisseurs, pour qui les placements dans le microcrédit, malgré ses crises, dégagent des marges plus importantes que des placements financiers classiques, en particulier depuis les turbulences globales de la finance après 2007 (Servet, 2015). C'est un argument que ne manquent pas de mettre en avant les intermédiaires financiers du secteur microfinancier en les qualifiant de « placements alternatifs ».

Pourtant, le poids du néolibéralisme ne s'arrête pas à un appui aux intérêts financiers dominants. L'idée selon laquelle les IMF doivent et peuvent s'autofinancer est, encore à ce jour, largement contredite par les faits. Si l'on tient compte de l'ensemble des subventions, directes et indirectes (y compris par des prêts à taux préférentiels), dont bénéficient les IMF, les puissances publiques sont mobilisées via plusieurs canaux au développement d'un secteur financier modelé par des lois et règlements, selon les principes de la concurrence. D'après les données du MIX Market, un quart d'entre elles seulement ne seraient pas subventionnées (Cull et al., 2009 ; D’Espallier et al., 2013). Une analyse plus fine montre que les IMF les plus aidées sont, d'abord et avant tout, celles à statut lucratif (et en premier lieu les banques), de plus grande taille, ciblant des emprunteurs plus aisés ${ }^{19}$, en priorité les hommes (Morduch, 2015). Les banques de microcrédit bénéficieraient de 241 dollars en moyenne par emprunteur servi, contre 117 dollars pour les ONG. Il s'agit toutefois là de données moyennes. On constate que des organisations à statut lucratif ont des montants moyens de crédits offerts inférieurs à ceux des coopératives d'épargne et de prêt, par exemple. Cela tient à ce que la durée de ces prêts est plus faible (quatre mois par exemple contre plus d'un an). Le volume total prêté sur une année à un même client est supérieur, mais le risque pris à consentir un prêt à cycle de remboursement court et pour un montant inférieur est plus faible. Rien d'étonnant par ailleurs à ce que ce type de prêts soit davantage utilisé pour la gestion courante des budgets domestiques, donc pour acquérir des biens de consommation. Toutefois, s'attacher uniquement à des critères financiers et commerciaux, tant pour évaluer les performances des organisations comme étant des entreprises pourvoyeuses de services financiers, que pour apprécier l'extension du secteur pour répondre aux besoins d'inclusion financière, tout comme tester selon ces mêmes critères la résilience à la montée des impayés, risque fort de laisser de côté l'essentiel de la transformation en cours : la contribution du microcrédit, dans ses formes dominantes actuelles ${ }^{20}$, à la gouvernance néolibérale des sociétés. L'endettement des ménages est un puissant moyen pour normer les comportements des populations, notamment lorsque cet endettement résulte de pratiques de démarchage et d'agressivité commerciale telles que celles décrites ici. Les contraintes du remboursement doivent faire intégrer aux ménages, plus encore avec l'éducation financière, la bonne

\footnotetext{
${ }^{19}$ D'après Jonathan Morduch (2015), si l'on prend le montant du prêt moyen rapporté au PIB par habitant, le ratio est de 0,5 pour les ONG, de 1,1 pour les Non Banking Financial Institutions et de 3,4 pour les banques. Même si le montant des prêts est une proxy très approximative du profil des emprunteurs, on constate tout de même un grand décalage de clientèle entre ONG et organisations à but lucratif.

${ }^{20}$ Nous avons développé par ailleurs une analyse des formes alternatives de microcrédit, tout en soulignant leurs difficultés à exister. Voir par exemple Isabelle Guérin (2015).
} 
gestion des ressources domestiques et des capacités de chacun de devenir une sorte d'entrepreneur de luimême. Il s'agit notamment d'inciter à réduire les dépenses jugées inutiles et à épargner pour investir productivement. Simultanément, les besoins de clients jugés moins solvables et plus risqués sont ignorés. En cela, le microcrédit est bien un dispositif du néolibéralisme, au sens donné à ce terme par Michel Foucault ${ }^{21}$.

\section{Bibliographie}

Afonso J., 2013, «Loans Officers and Preventing Overindebtedness - The Case of Banco Adopem », Research and Policy Briefs, $\mathrm{n}^{\circ}$, Nogent-sur-Marne, IRD/Microfinance in Crisis, http://www.microfinance-in-crisis.org/wp-content/uploads/Research-and-Policy-Brief 8.pdf (janvier 2016).

Banerjee A., Moll B., 2010, «Why Does Misallocation Persist? », American Economic Journal: Macroeconomics, vol. 2 , n 1, pp. 189-206.

Banerjee A., Karlan D., Zinman J., 2015, « Six Randomized Evaluations of Microcredit: Introduction and Further Steps », American Economic Journal: Applied Economics, vol. 7, $\mathrm{n}^{\circ}$ 1, pp. 1-21, http://economics.mit.edu/files/10475 (janvier 2016).

Bédécarrats F., 2013, La Microfinance. Entre utilité sociale et rentabilité financière, Paris, L'Harmattan.

Bédécarrats F., Guérin I., Roubaud F., 2015, « The Gold Standard for Randomised Evaluations: From

Discussion of Method to Political Economy, Working Paper, $\mathrm{n}^{\circ}$ 2015-01, http://en.dial.ird.fr/content/download/210000/3259473/version/3/file/DT+2015-

01+B $\% \mathrm{C} 3 \% \mathrm{~A} 9 \mathrm{~d} \% \mathrm{C} 3 \% \mathrm{~A} 9$ carrats+-+Gu\% $\% \mathrm{C} 3 \% \mathrm{~A} 9$ rin+-+Roubaud+-+EN.pdf (janvier 2016). Bernard T., Delarue J., Naudet J.-D. (2012), « Impact evaluations: a tool for accountability? Lessons from experience at Agence Française de Développement ", Journal of Development Effectiveness, 4 (2), pp. 314-327.

Berndt C., 2015, « Behavioral Economics, Experimentalism and the Marketization of Development », Economy and Society, academia.edu, http://www.academia.edu/17249645/Berndt C. 2015 Behavioural economics experimentalism and the marketization of development Economy and Society online first (janvier 2016)

CGAP, 2013, «Ce que la crise marocaine peut nous apprendre », Brief Note, Washington, Consultative Group to Assist the Poor.

Collins D., Morduch J., Rutherford S. et al., 2010, Porfolios of the Poor: How the World's Poor Live on $\$ 2$ a Day, Princeton, Princeton University Press, p. 180.

21 Ce dispositif étant entendu comme un ensemble stratégique de discours, d'énoncés savants, de mesures administratives, de décisions réglementaires et de propositions de toute nature visant à manipuler les humains pour les amener non pas simplement à se soumettre à l'ordre dominant mais à en devenir utile en tant que leur coproducteur (Foucault, 2001). 
Cull R., Demirgüç-Kunt A., Morduch J., 2009, « Microfinance Meets the Market », Journal of Economic $\begin{array}{llllll}\text { Perspectives, } & \text { vol. } & 23 & \mathrm{n}^{\circ} & 1, & \text { pp. }\end{array}$ https://www.aeaweb.org/articles.php?doi=10.1257/jep.23.1.167 (janvier 2016).

D’Espallier B., Hudon M., Szafarz A., 2013, « Unsubsidized Microfinance Institutions », Economics Letters, vol. 120, n 2, pp. 174-176.

D’Espallier B., Labie M. et Louis Ph., 2015, « Microcredit Crises and Unsustainable Growth: A Management Perspective », in Guérin I., Labie M. et Servet J.-M. (ed.), The Crises of Microcredit, Londres, Zed Books.

Foucault M., 2001, Dits et écrits, tome 2 : 1976-1988, Paris, Gallimard, pp. 299-301.

Foucault M., 2004, Naissance de la biopolitique : Cours an Collège de France (1978-1979), Paris, Le Seuil, pp. 120 121, 231-232, 248, 274.

Fouillet C., Morvant-Roux S., 2015, « State Building in India and Mexico: Is Financial Inclusion a Missing Link? », 27 e conférence de la Society for the Advancement of Socio-Economics, London School of Economics and Political Science, 1-4 juillet.

González C., Servet J.-M., 2014, « Pourquoi le microcrédit en République dominicaine a échappé à la crise », Rapport de mission, Nogent-sur-Marne, Microfinance in Crises/BEI.

Guérin I., 2014, «Juggling with Debt, Social Ties, and Values. The Everyday Use of Microcredit in Rural South India ", Current Anthropology, vol. 55, n S9, pp. S40-S50, http://www.jstor.org/stable/10.1086/675929?seq=1\#page scan tab contents (janvier 2016).

Guérin I., Roesch M., Venkatasubramanian G., D’Espallier B., 2012, «Credit from Whom and for What? Diversity of Borrowing Sources and Uses in Rural South-India », Journal of International Development, $\mathrm{n}^{\circ} 24$, pp. S122-S137.

Guérin I., 2015, La microfinance et ses dérives. Émanciper, discipliner ou exploiter?, Paris, Demopolis/IRD.

Guérin I., D’Espallier B., Venkatasubramanian G., 2013, « Debt in Rural South India: Fragmentation, Social Regulation and Discrimination », Journal of Development Studies, vol. 49, $\mathrm{n}^{\circ}$ 9, pp. 1155-1171.

Guérin I., Labie M., Servet J.-M. (ed.), 2015, The Crises of Microcredit, Londres, Zed Books.

Guérin I., Roesch M., Venkatasubramanian G. et al., 2009, « Microfinance, endettement et surendettement. Une étude de cas en Inde du Sud ", Revue Tiers Monde, $\mathrm{n}^{\circ}$ 197, pp. 131-146, http://www.cairn.info/revue-tiers-monde-2009-1-page-131.htm (janvier 2016).

Hes T., Poledňáková A., 2013, « Correction of the Claim for Microfinance Market of 1.5 Billion Clients », International Letters of Social and Humanistic Sciences, $\mathrm{n}^{\circ} 2$, pp. 18-31, http://www.scipress.com/ILSHS.2.18 (janvier 2016).

IFC, 2014, Sortie de la crise du secteur de la microfinance au Maroc : des interventions opportunes et efficaces, Le Caire, IFC/Banque mondiale, http://www.ifc.org/wps/wcm/connect/f45be1004785101ca0e9f5299ede9589/IFC+Morocco+ MicroFinance + Crisis + report.French.pdf?MOD=AJPERES (janvier 2016). 
Johnson S., Williams R., 2013, «The political Economy of Financial Inclusion: Working with Governments on Market Development », Bath papers in international development and well-being, $\mathrm{n}^{\circ} 23$, CDS/University of Bath, http://www.bath.ac.uk/cds/publications/bpd23.pdf (janvier 2016).

Kar S., 2013, « Recovering Debts: Microfinance Loan Officers and the Work of "Proxy-Creditors” in India», American Ethnologist, vol. 40, $\mathrm{n}^{\circ}$ 3, pp. 480-493.

Karlan D., Appel J., 2011, More than Good Intentions: How a New Economics is Helping to Solve Global Poverty, New York, Dutton.

Landy F., 2001, « La libéralisation économique en Inde : inflexion ou rupture ? », Revue Tiers Monde, t. 42, n 165, pp. 9-16, http://www.persee.fr/doc/tiers 1293-8882 2001 num 421651466 (janvier 2016).

Lascelles D., Mendelson S., 2012, Microfinance Banana Skins 2012: Staying Relevant, Londres, Centre for the Study of Financial Innovation, https://www.cgap.org/sites/default/files/CGAP-MicrofinanceBanana-Skins-Jul-2012.pdf (janvier 2016).

Lascelles D., Mendelson S., Rozas D., 2014, Microfinance Banana Skins 2014: Facing Reality, Londres, Centre for the Study of Financial Innovation, pp. 1-8, https://centerforfinancialinclusionblog. files.wordpress.com/2014/07/2014 microfinance bana na skins.pdf (janvier 2016).

Lazzarato M., 2015, Governing by Debt, Cambridge, MIT Press/Semiotext(e).

Morduch J., 2015, "Another look at microfinance subsidies », 4th European Research Conference on Microfinance, Université de Genève, 2 juin.

Morvant-Roux S., Roesch M., 2015, « The Social Credibility of Microcredit in Morocco after the Default Crisis », in Guérin I., Labie M., Servet J.-M. (ed.), The Crises of Microcredit, Londres, Zed Books.

Morvant-Roux S., Afonso J., Forcella D. et al., 2015, « How good repayment performances can harm borrowers: Evidence from the Dominican Republic », in Guérin I., Labie M., Servet J.-M. (ed.), The Crises of Microcredit, Londres, Zed Books, pp. 92-112.

Morvant-Roux S., Guérin I., Roesch M. et al., 2014, « Adding Value to Randomization with Qualitative Analysis: The Case of Microcredit in Rural Morocco », World Development, n 56, pp. 302-312.

Picherit D., 2015, "When Microfinance Collapses: Development and Politics in Andhra Pradesh », in Guérin I., Labie M., Servet J.-M. (ed.), The Crises of Microcredit, Londres, Zed Books, pp. 170-186.

Planet Rating, 2013, Mimosa, Microfinance Index of Market Outreach and Saturation, Paris, Fondation PlaNet Finance, p.

15 , http://www.planetrating.com/userfiles/file/MIMOSA\%201_0 final\%20110313.pdf (janvier 2016).

Quentin A., Guérin I., 2013, « La randomisation à l'épreuve du terrain. L'exemple du projet de microassurance Sky au Cambodge ", Revue Tiers Monde, $\mathrm{n}^{\circ}$ 213, pp. 179-200, https://www.cairn.info/load pdf.php?download=1\&ID ARTICLE=RTM 2130179 (janvier 2016). 
Reed L. R., Maes J. P., 2014, État de la campagne du sommet du microcrédit, Rapport, Washington, Campagne du Sommet $\mathrm{du}$ microcrédit, http://www.microcreditsummit.org/uploads/resource/document/web socr2012 french 19952.pdf (janvier 2016).

Servet J.-M., 2006, Banquiers aux pieds nus. La microfinance, Paris, Éd. Odile Jacob.

Servet J.-M., 2011, «La crise du microcrédit en Andhra Pradesh (Inde) », Revue Tiers Monde, n² 207, pp. 4359, http://www.cairn.info/revue-tiers-monde-2011-3-page-43.htm (Janvier 2016).

Servet J.-M., 2015, La V raie Révolution du microcrédit, Paris, Éd. Odile Jacob.

Stiglitz J. et Weiss A., 1981, «Credit rationing in markets with imperfect information », American Economic Review, vol. 71, n 3, pp. 393-410.

Taylor M., 2011, «“Freedom from Poverty is Not for Free”: Rural Development and the Microfinance Crisis in Andhra Pradesh, India ", Journal of Agrarian Change, vol. 11, $n^{\circ} 4$, pp. 484-504.

Waterfield C., 2015, «MFTransparency is Dead... What Does That Mean for Pricing Transparency? », mfttransparency.org, http://www.mftransparency.org/homeslide/mftransparency-deadmean-pricing-transparency/ (janvier 2016).

Woller G., Dunford C. et Warner W., 1999, «Where to microfinance? », International Journal of Economic Development, vol. 1, n 1, pp. 29-64. 